\title{
Size and shape optimization of aluminum tubes with GFRP honeycomb reinforcements for crashworthy aircraft structures
}

\author{
J. Paz, J. Díaz*, L. Romera, M. Costas \\ Structural Mechanics group, School of Civil Engineering, Universidade da Coruña, Campus de Elviña \\ 15071 A Coruña, Spain
}

\begin{abstract}
Crashworthiness optimization of aircraft and automotive structures has become one the main research targets for their respective leading industries. The following research proposes a new design of an aircraft's vertical strut. The design consists of a hollow aluminum square tube with a glass-fiber reinforced polymer honeycomb-shaped inner structure. Size and shape surrogate-based optimization techniques are used, with the thicknesses of both materials, cell size and cell shape as design variables. The objective function chosen for the single-objective optimization is the specific energy absorption, while the metrics for the multi-objective optimization are the peak force, mass, absorbed energy and the specific energy absorption. An improvement of $22 \%$ of the specific energy absorption with low peak force values is obtained from the singleobjective optimization by significantly changing all design variables. Two Pareto fronts have been obtained from the multi-objective optimization confronting, the specific energy absorption against the peak force and the mass against the energy absorbed. When compared to the baseline model, the optimized models show substantial improvement, increasing the specific energy absorption by $65 \%$ or reducing the peak force by over $55 \%$. It has been observed an important effect of the cell shape on the model's performance.
\end{abstract}

Keywords: crashworthiness, multi-objective optimization, surrogate methods, honeycomb structure, GFRP

\section{Introduction}

Structural optimization is one of the key research targets for the aircraft and automotive design industries. Particularly, since the 1970s, structural design refinement of both civil and military aircrafts is aimed towards reduction of the mass, and consequently, an increase of the aircraft's payload. However, this mass reduction also needs

\footnotetext{
*Corresponding author. Tel: +34 881011 407; Fax: +34 981167170

Email addresses: javier.paz.mendez@udc.es (J. Paz), jdiaz@udc.es (J. Díaz), lromera@udc.es (L. Romera), miguel.costas@udc.es (M. Costas)
} 
to consider the aircraft's crashworthiness, which takes into account the crash survivability during an impact.

Crashworthiness is defined not only as a structural or material characteristic but also as a combination of both of them, so that a structure exhibits an outstanding crash-resistance and, additionally, exceptional energy-absorption capabilities. Therefore, forces and accelerations transmitted to the passengers need to be kept under a certain value, while maintaining the necessary living space after a crash. As an answer to these necessities, diverse standards have been established such as the requirements by the Federal Aviation Administration (FAA) or the Joint Airworthiness Requirements (JAR) [1]. Crashworthiness-wise improvement of the landing gear, main fuselage sections, cabin layout and occupant seat systems has noticeably increased the survival probabilities and lessened passenger injuries after a crash.

In order to measure its crashworthiness, an aircraft is tested under different situations, including bird strike simulations [2], impact on wings [3], and water ditching and crash landing scenarios on solid grounds $[4,5]$. These last situations, modeled with a vertical drop test, are commonly employed by structural designers to ensure the desired craft's performance.

Enhancing the fuselage of an aircraft according to the crashworthiness criteria requires modifying its failure behavior, monitoring the acceleration characteristics and increasing the structure's energy absorption capabilities. The main deformation area during an impact or hard landing is located under the cabin floor, where three primary structures absorb most of the energy generated during the crash [6]: the sub-cargo area, the aircraft's frame and the vertical struts (fig. 1).

The aircraft's sub-cargo region is the first to collide with the ground, therefore being the major area crushed during a potentially survivable crash accident. Many authors are drawing their efforts towards the optimization of this section, having tried several alternatives to improve its performance. The use of composite sine-wave beams in the sub-floor structure [7], evenly spaced blocks of crushable foam [8], and folded core [9] and honeycomb structures [10] have recently proved their remarkable capabilities ebbing away the abrupt deceleration peaks suffered by passengers.

After the impact affects the sub-floor, it soon reaches the circumferential frame. The approaches followed to improve this area range from integrating additional absorbers and plastic hinges which translate into a progressive failure scheme [11] to the implementation of corrugated foam sandwich panels in the frame [12].

Lastly, the vertical struts, designed to function as energy absorbers without altering the main airframe, are also heavily loaded during a crash. Since they connect the passenger cabin floor and the lower part of the frame, they act as vertical support in the middle-to-large size aircraft range [13]. During an impact, these vertical tube-like structures are subjected to axially-dominated compressive loads. Its effect on the crashworthiness of the aircraft is greatly dependent on their overall stiffness [14, 15]: while a rigid strut helps the lower part of the fuselage dissipate more energy, the cabin floor would endure more if flexible struts are fitted. However, and despite their acute effect on the aircraft's performance, no study conducted up to date considers a continuous trade-off between the energy absorbed by the strut and its rigidity.

In order to effectively integrate the strut in the aircraft, several approaches have been already proposed. Ren and Xiang [16] studied different configurations and po- 


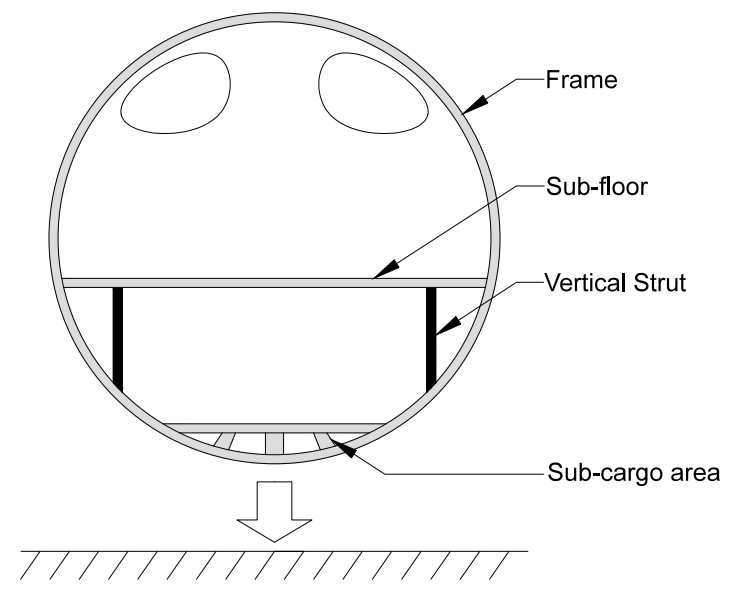

Figure 1: Schematics of an aircraft fuselage drop test for crashworthiness evaluation.

sitioning of the struts, yielding useful considerations on their energy absorption, acceleration characteristics and failure behavior. Heimbs et al. [17] extensively tested a representative strut with an integrated lightweight composite crash absorber. The model was subjected to static, dynamic, and fatigue load cases, also considering the effects of axial or oblique impact conditions. Also, Ren and Xiang [18] studied the implementation of triggers in quadrangular struts, forcing failure of the structure after reaching a certain stress value, thus modifying the failure behavior, but without significantly harming the energy absorption capabilities. Zou et al. [19] explored the response of the lower half of the fuselage section during a crash in relation to the angle of the vertical struts and their rigidity. This parameter study performed constitutes a solid starting point for future continuous optimization of both the mechanical characteristics of the strut and the angle in which it is attached.

On the other hand, and in order to improve the performance of metallic struts, techniques have also evolved towards the use of composite materials, gaining importance rapidly in the field during the last two decades. The concept is based on combining two or more materials in order to obtain a better structural response than with them separately. A hollow tubular metallic structure filled with a core of another material complementing the tube's response is a typical example recurrently used. The inner part has been thoroughly looked into, and by changing its materials and shape, the response of the combined outer and inner structures is enhanced. By doing so, the ductile collapse process of the metal is constrained by the inner material, acting as a core bracing affect. Consequently, and as Song et al. [20] have identified, higher values of the total energy absorbed are obtained. Furthermore, this also translates into a more effective exploitation of both the outer and inner materials' mechanical properties, as the energy absorbed by the complete structure is greater than the sum of the energy absorbed by both parts detached [21].

Concerning these inner reinforcements, their shapes can differ substantially, ranging from tight-compact to honeycomb-like structures, including complex geometries or 
cores of laminates. While all of them improve the performance of the structure [22], the honeycomb has proved to be excellent in bettering the response of the complete structure [23, 24, 25]. Miller et al. [26] proved that a modified honeycomb cell resembling an hourglass shape can improve the buckling strength of the inner reinforcement under axial load cases, while Xue and Hutchinson [27] also studied the behavior of square sandwich panels. Nevertheless, no significant further research was made on the matter, opening the possibility for a reliable cell shape optimization of honeycomb cores under axial loads.

In this investigation, the traditional strut improvement is taken one step further. A standard thin-walled aluminum strut is filled with a glass-fiber reinforced polymer (GFRP) honeycomb structure, only to be later on modeled and analyzed using nonlinear finite element analyses. Size and shape optimization of the honeycomb structure is newly performed, as no significant contemporary research has been done on the matter.

By optimizing the tube's wall thickness and the honeycomb's cell shape, size and wall thickness, the most advantageous overall structural shape shall be obtained. The criteria used for the optimization process considers the energy-absorption performance of the strut as well as the survivability of passengers. Due to the large computational cost of running each analysis, a surrogate-based optimization approach is used. After the optimization process, the results shall be once again tested to prove the accuracy of the methods used and the effectiveness of the strut as an energy-absorption structure. Finally, the improved design is compared to the baseline model, thus being able to quantify the improvement of the optimized model obtained.

\section{Vertical strut design}

This research focuses on an isolated standard vertical strut used in aircraft structures. The specimen is composed of two separate elements: an outer metallic tube filled with a honeycomb-like glass-fiber reinforced polyamide structure. This part has a cell distribution with variable size and shape of the cells.

\subsection{Materials}

The outer part is made of an AA7075-T651 aluminum alloy; while the inner structure is made of the glass-fiber reinforced polyamide Ultramid A3WG10 BK00564 (BASF).

The aluminum strain-stress curves follow the modified Johnson-Cook strain-rate sensitive model proposed by Børvik et al. [28]. Given the nature of this investigation, the temperature dependency is ruled out of the equation. Hence, the constitutive equation of the Mises plasticity model used to simulate the aluminum is defined as

$$
\sigma_{y}=\left(A+B p^{n}\right)\left(1+\frac{\dot{p}}{\dot{p_{0}}}\right)^{C}
$$

where $A$ is the material's yielding stress, $B$ is the hardening law's amplitude modifier,

$p$ is the equivalent plastic strain, $\frac{\dot{p}}{\dot{p}_{0}}$ is the dimensionless plastic strain rate, and $C$ is 
the strain-rate's dependency modifier. All material and model parameter values, taken from the work of Børvik et al. [29], are detailed in table 1. Figure 2 provides the stress-strain curve for a model subjected to a strain rate of $0.035 \mathrm{~s}^{-1}$.

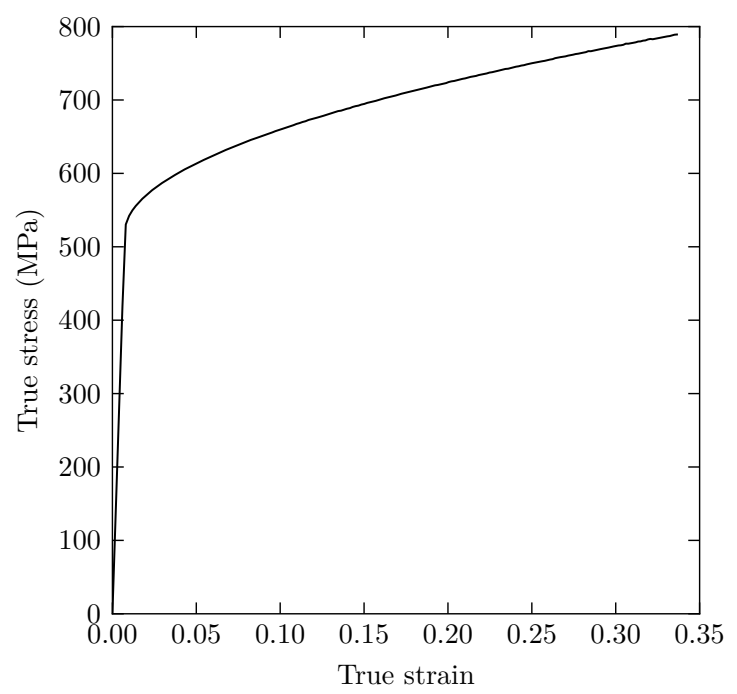

Figure 2: Stress-strain curve of the AA7075-T651 aluminum at a strain rate of $0.035 \mathrm{~s}^{-1}$.

\begin{tabular}{cccccccc}
\hline$E$ & $v$ & $\rho$ & $\mathrm{A}$ & $\mathrm{B}$ & $\mathrm{n}$ & $\mathrm{C}$ & $\dot{p_{0}}$ \\
\hline $70 \mathrm{GPa}$ & 0.3 & $27.7 \mathrm{kN} / \mathrm{m}^{3}$ & $520 \mathrm{MPa}$ & $477 \mathrm{MPa}$ & 0.52 & 0.001 & 0.0005 \\
\hline
\end{tabular}

Table 1: AA7075-T651 aluminum properties and Mises plasticity model values for eq. (1). Taken from Børvik et al. [29].

The material constitutive model has been calibrated after the data recorded from tensile tests. The expression shown in eq. (2) was adjusted with its four parameters to fit the experimental data. The density and the Poisson's modulus have been extracted from the manufacturer's data sheets [30]. All data is listed in table 2.

$$
\sigma_{y}=\sigma_{0}+\left[\sum_{j=1}^{k} Q_{j}\left(1-e^{-b_{j} p}\right)\right]
$$

where $Q_{j}, b_{j}$ and $\sigma_{0}$ are material constants, and $k$ is the number of terms used to approximate the material's response. For this material, $k=2$, with a true strain at failure of $2.38 \%$. Figure 3 provides the numerical and experimantal stress-strain curves for the GFRP model used. 


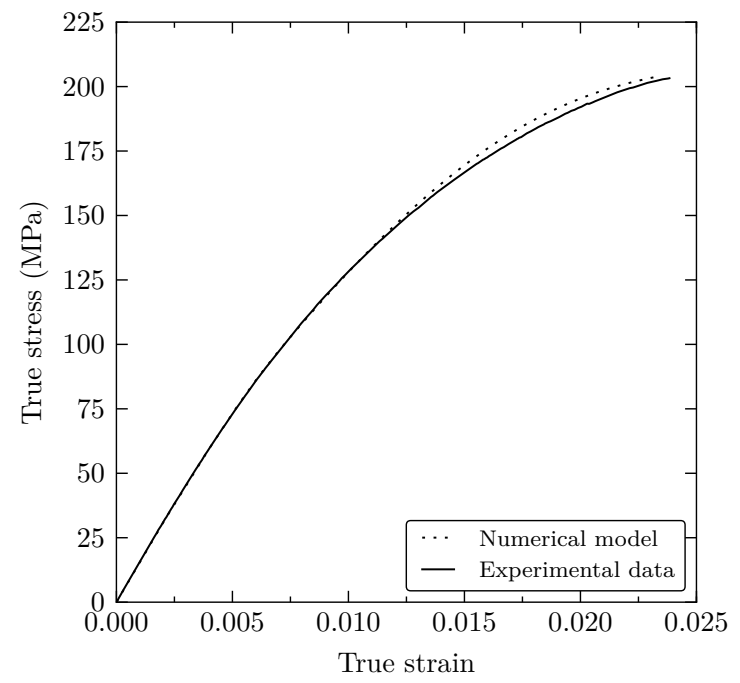

Figure 3: Numerical and experimental tensile stress-strain curves of the Ultramid A3WG10 BK00564 up to fracture at a strain rate of $3.25 \times 10^{-4} \mathrm{~s}^{-1}$

\begin{tabular}{cccccccc}
\hline $\mathrm{E}$ & $v$ & $\rho$ & $\sigma_{0}$ & $Q_{1}$ & $Q_{2}$ & $b_{1}$ & $b_{2}$ \\
\hline $15.4826 \mathrm{GPa}$ & 0.4 & $15.15 \mathrm{kN} / \mathrm{m}^{3}$ & $25 \mathrm{MPa}$ & $124.86 \mathrm{MPa}$ & $44.46 \mathrm{MPa}$ & 315.89 & 5748.46 \\
\hline
\end{tabular}

Table 2: Material properties of Ultramid A3WG10 BK00564.

\subsection{Design variables and objective functions}

Three design parameters and four design variables define the geometrical configuration of the specimen. The design parameters are the height of the tube $(500 \mathrm{~mm})$, the edge-length of the tube $(100 \mathrm{~mm})$, and the height of the inner reinforcement (480 $\mathrm{mm})$. The 20-millimeter height difference between the inner reinforcement and the tube assures an offset in the initial force peaks during the crushing process, resulting in a lower combined peak force [21].

On the other hand, the design variables chosen are the thickness of the aluminum plates (T1), the thickness of the GFRP honeycomb reinforcement (T2), the half-length of a single honeycomb cell (L1), and the honeycomb cell shape modifier (S) (figs. 4 and 5). This last variable is responsible for the honeycomb's shape optimization, and it is defined as

$$
S=\frac{L 2}{L 1}
$$

The effect of the shape modifier variable is shown in fig. 5. For a value of $S=0$ (fig. 5a), the honeycomb cell is a regular hexagon. As the value of $\mathrm{S}$ increases, the cell 
shape tends to a rectangle with a length to width ratio of $1.73: 1$, achieved for $S=0.5$ (fig. 5b). For values greater than $S=0.5$, and with an upper boundary of $S=0.75$, the cell shape resembles that of an hourglass, as shown in fig. $5 \mathrm{c}$.

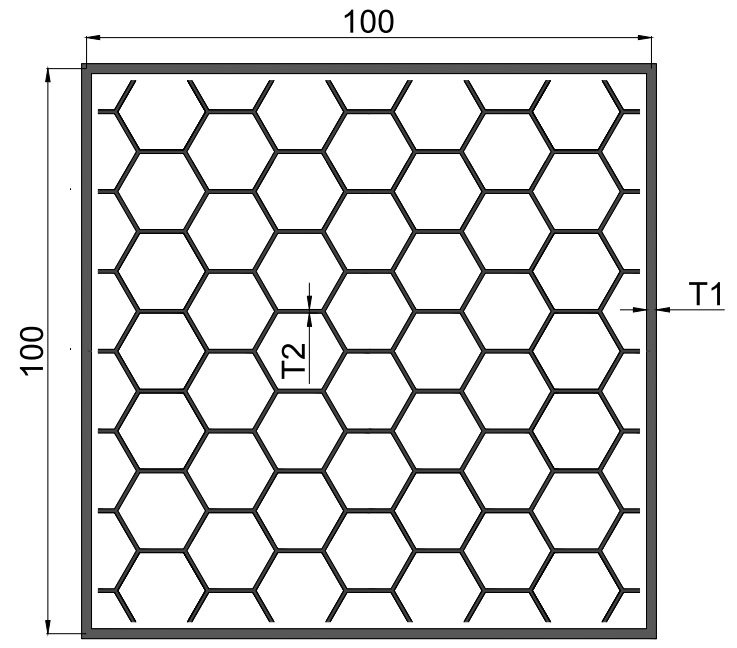

Figure 4: Top view of the specimen. All values in millimeters.

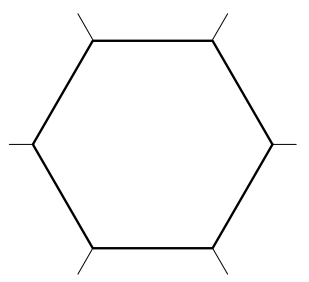

(a) Regular hexagon. $S=0$

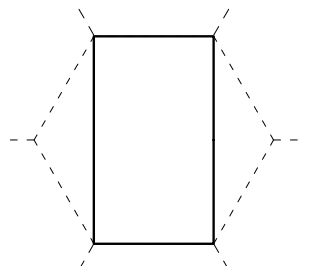

(b) Rectangle. $\mathrm{S}=0.5$

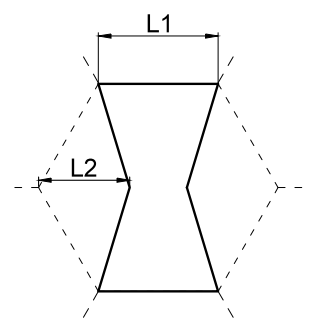

(c) Hourglass shape.

$0.5 ; \mathrm{S} ; 0.75$

Figure 5: Different honeycomb cell shape configurations.

The initial values and upper and lower bounds of all design variables, as well as the description of which part they belong to, are listed in table 3 .

Concerning the objective functions, different options have been considered. Simple metrics, such as the total energy absorbed or the mass of the structure, are usually taken into account. However, in an attempt to improve lightness and robustness at a time, the maximization of the specific energy absorption ratio (SEA) has also been considered. Furthermore, and for the benefit of the passengers of the aircraft and its integrity, the peak force values are minimized. By doing so, the survivability odds increase, as well as reducing the damage to the craft and occupants. The four metrics selected are the absorbed energy $\left(E_{a}\right)$, the mass of the specimen $(m)$, the specific energy absorption $(S E A)$ and the maximum force suffered during the crushing $\left(P_{p e a k}\right)$. 


\begin{tabular}{lcccc}
\hline & & \multicolumn{2}{c}{ Bounds } & \\
\cline { 3 - 4 } Part & Variable & Lower & Upper & Initial Value \\
\hline Tube & T1 & 0.87 & 2.44 & 1.50 \\
\hline \multirow{2}{*}{ Honeycomb } & T2 & 1.00 & 3.00 & 1.50 \\
& L1 & 5.88 & 14.30 & 10.00 \\
& $\mathrm{~S}$ & 0.00 & 0.75 & 0.00 \\
\hline
\end{tabular}

Table 3: Bounds and initial values of design variables. All dimensions in millimeters.

All responses except the mass have been obtained from the force-displacement curves calculated by a finite element analysis. These data is post-processed with a standard SAE 600 filter [31], as recommended by Huang [32], removing the highfrequency noise with a cutoff frequency of $1000 \mathrm{~Hz}$.

$E_{a}$ is given by the filtered curves as

$$
E_{a}=\int_{0}^{\delta_{\max }} F(\delta) d \delta
$$

where $\delta_{\max }$ is the maximum crushing distance before bottoming-out and $F(\delta)$ is the crushing force obtained at a crushing length of $\delta$. The $S E A$ is defined as the ratio between $E_{a}$ and $m$

$$
S E A=\frac{E_{a}}{m},
$$

and the peak load, $P_{\text {peak }}$, is obtained as

$$
P_{\text {peak }}=\max \left\{F(\delta) \forall \delta \in\left[0, \delta_{\max }\right]\right\}
$$

The reason for choosing these objective functions derives from the necessity of a reliable crashworthiness optimization. As in any multi-objective optimization, the objective functions need to relate to different aspects of the model's capabilities. By maximizing the $E_{a}$, structural deformations are localized in the strut area, since less energy needs to be absorbed by other surrounding structures. Furthermore, reducing the element's mass decreases the building cost and fuel consumption as well. Nevertheless, single-objective optimization requires only one metric, and maximizing the $S E A$ ratio would entail both a maximization of the $E_{a}$ and a minimization of the specimen's mass. However, even though they provide important information about the model's responses, metrics that derive from ratios tend to have noisier behaviors, thus also justifying the use of the first two metrics as objective functions. Finally, the occupant's safety is always considered by reducing the maximum deceleration suffered at the end of the tube opposite to the impacting mass' strike, $P_{\text {peak }}$. 


\section{Surrogate model and optimization methods}

Due to the large computational requirements of this investigation based on finite element modeling and analysis, the use of surrogate models is imperative. These methods have proved very effective when relating the impact responses to the design variables in highly non-linear optimization problems. After sampling the workspace at the sampling points desired, a surrogate function is fitted, so it can later save significant computational time being the subject of the optimization process. In the 1990s, Yamazaki and Han [33] began using surrogate-based crashworthiness optimization applied to circular and square tubes. Later on, and given the problem size increase and the capabilities of these methods, Gu et al. [34] evaluated the reliability and robustness of different surrogate models. Nowadays, these methods are very commonly used, given the size and complexity increase of recent models.

Surrogate-based methods replace the complex and unsteady objective functions from the original model, $f_{i}$, by more tractable functions, $\hat{f}_{i}$, which can approximate these original model's responses. By doing so, the computational resources are aimed towards building a robust surrogate model rather than towards performing time-consuming evaluations of each model during the optimization process. Therefore, once the surrogate model is built, evaluating its response, considering any values of the design variables within their boundaries, verges on immediacy, replacing the lengthy evaluation of a complete model.

In order to obtain an efficiently-built and accurate surrogate model, the number of data points sampled and the resemblance between the original and surrogate models are to be reckoned with. Hence, the sampling of the design workspace shall be performed with the minimum number of points $n$ that yield the required precision, but without requiring excessive computational resources. The latin hypercube sampling (LHS) [35] is an efficient sampling strategy for such a purpose, as it creates a set of data points with no overlapping projections onto each variable axis, increasing the dispersion of the sampling.

Once the $n$ points have been computed, the next stage fits a function adjusting to these points. A considerable number of strategies are available for the task, such as Gaussian processes or multivariate adaptive regression splines (MARS), but for this model, the moving least squares (MLS) technique was chosen.

The MLS regression model fits polynomials as the surrogate model according to:

$$
\hat{f}(\boldsymbol{x})=\sum_{m=1}^{M} c_{m} B_{m}(\boldsymbol{x})
$$

where $B_{m}$ are the polynomial basis functions, $c_{m}$ are the coefficients of the functions, $M$ is the number of functions and $\boldsymbol{x}$ is the design variables' vector. To adjust the $c_{m}$ coefficient, the sum of the squared residuals - the differences between the surrogate model and the real values at the $n$ sampling points - is minimized. Each residual is also assigned a point-specific weight $\omega_{h}$ that considers its relevance to the overall model as follows:

$$
\sum_{h=1}^{n} \omega_{h}\left(\left\|\hat{f}\left(\boldsymbol{x}_{h}\right)-f\left(\boldsymbol{x}_{h}\right)\right\|\right)
$$


This method and its working principles are more thoroughly explained by Lancaster and Salkauskas [36].

Once the surrogate model is built, the last stage consists in the optimization of the model. The process resorts to two types of optimization: size optimization and shape optimization. Size optimization determines the best thickness or dimensions of a structure under certain load conditions and constraints in order to improve its performance. During shape optimization, however, the spatial configuration of a structure, or group of structures, morphs until an optimal distribution is obtained, also complying with a series of spatial constraints defined beforehand.

A single-objective genetic algorithm (SOGA) from the JEGA library [37] is used to obtain valuable knowledge of the specimen's behavior. However, in order to integrate the optimization of all objective functions in a single and continuous process, multiobjective optimization is used. The multi-objective genetic algorithm (MOGA), also from the JEGA library, performs Pareto optimization while supporting general constraints and a combination of discrete and continuous variables. Both methods extrapolate the theory of the survival of the fittest to the mathematical field. On that account, each design variable constitutes a chromosome, and their respective digits are treated as genes. However, and despite its robustness for these noisy functions, the computing time can be significantly high and the optimum obtained may not be guaranteed to be the global optimum. Thus, using crossover and mutation techniques and empirically adjusting the parameters listed in table 4 is of vital importance, as they help evade local minima and improve the convergence of the algorithm.

The optimization results provided by the MOGA include the closest points to the utopia point, defined as the point which would minimize all objective functions, and the Pareto frontier. The Pareto front shows the points which cannot better one of the objective function's response without harming others, thus representing a trade-off between the target responses considered in the investigation.

\begin{tabular}{lr}
\hline Parameter & Value \\
\hline Population size & 500 \\
Offset normal mutation rate & 0.8 \\
Two-point crossover probability & 0.8 \\
Elitism & 50 \\
Maximum allowed individuals & $10^{5}$ \\
\hline
\end{tabular}

Table 4: Configuration parameters for the JEGA library optimization algorithms.

\subsection{Analysis settings}

The specimen and its analysis conditions are implemented in a FEA software. The model is meshed according to its size, the mechanical behavior of the materials, and the computational resources at hand. Hence, for the aluminum, a metal with a considerable ductile behavior, the folding process is accurately discerned with a mesh size of $4 \mathrm{~mm}$. However, due to the GFRP's elastic damage scheme, the honeycomb's mesh size is 
smaller and dependent on the honeycomb cell size, taking values between $1.5 \mathrm{~mm}$ and $3 \mathrm{~mm}$, so that every cell has between two and six elements. Two three-dimensional cuts of the meshed specimen are shown in figs. $6 \mathrm{a}$ and $6 \mathrm{~b}$, namely, the former with a regular hexagonal honeycomb shape and the latter with a modified version.

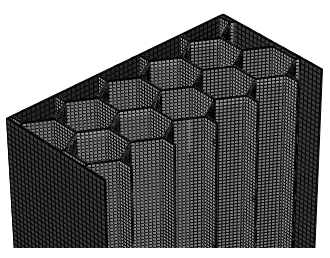

(a) Regular hexagonal honeycomb. $\mathrm{S}=0$

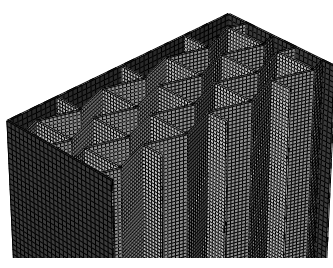

(b) Modified hexagonal honeycomb. $\mathrm{S}=0.5$

Figure 6: Three-dimensional cut of the finite element mesh of the specimen.

Concerning the finite element simulation settings, they attempt to replicate the conditions in which the structure would be crushed, had it been implemented in a full-scale fuselage. The first step of the process forces a two-millimeter triggering on the upper edges of the aluminum tube in order to obtain a regular collapse mode and reduce the initial peak force in the subsequent steps. Figures $7 \mathrm{a}$ and $7 \mathrm{~b}$ show the difference between two identical specimens with the only the non-triggered and triggered collapse schemes, with this last one having a more organized fold distribution. The initial peak force of the triggered specimen is also $31 \%$ lower (fig. 8).

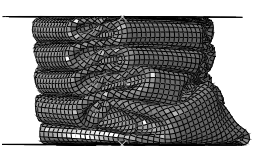

(a) Non-triggered collapse scheme.

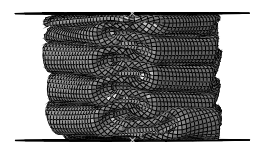

(b) Triggered collapse scheme.

Figure 7: Comparison of the collapse modes.

To determine the impact velocity, the test conditions simulated by other authors are taken into account (table 5). Its diversity is relatively moderate, with values often between $6.5 \mathrm{~m} / \mathrm{s}$ and $9.5 \mathrm{~m} / \mathrm{s}$. As for this experiment, the specimen is crushed a total length of $0.4 \mathrm{~m}$ at a constant velocity of $10 \mathrm{~m} / \mathrm{s}$.

The sampling consists of 500 data points, sufficient to accurately model the design variables' feasible workspace. Each data point constitutes the analysis of a specific model, which is run with the explicit module of the Abaqus 6.13 FEA package [42]. The problems were first divided into 8 domains - each of them computed in separate processors with 3000 megabytes of RAM - since a previous study revealed this configuration gives the best trade-off between the consumed computational resources and accuracy of the analysis. Every evaluation is dissected into 600 time steps, in which the software records the corresponding output variables and the force-strain curves. Afterwards, the surrogate-based optimization procedure and the optimization algorithms, 


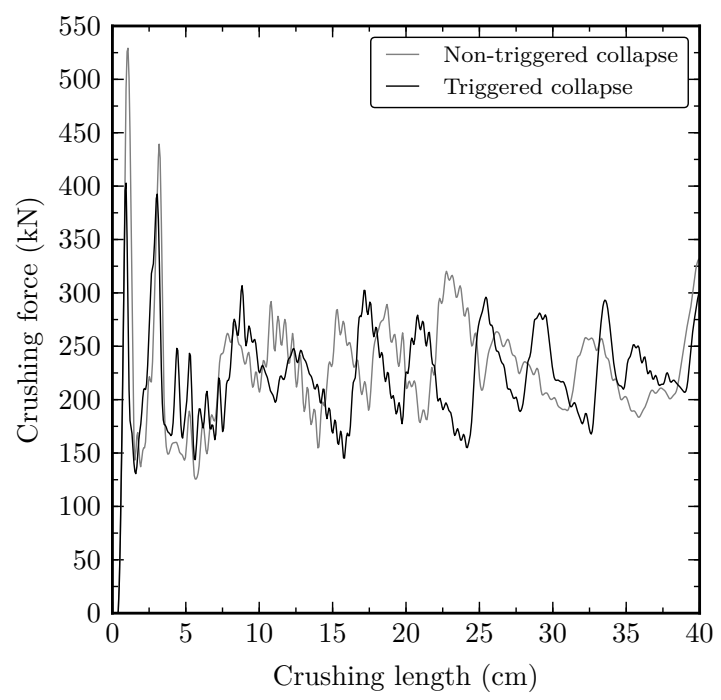

Figure 8: Force - displacement curves of the non-triggered and triggered collapse schemes.

implemented via DAKOTA framework in its version 6.1.0 [43], are executed. All analyses have been run in a high performance computing (HPC) cluster with a theoretical peak performance of 7.6 TFLOP's.

\section{Results}

\subsection{Initial results}

Results and their validity closely depend on the accuracy of the surrogate model. The $R^{2}$ and the root mean squared error (RMSE) metrics have been used to assess the accuracy of two surrogate models, with the RMSE being computed under a 10fold cross-validation technique. Table 6 compares a second order moving least squares model and a multivariate adaptive regression splines model with a maximum of 74 basis functions. The results show a better overall performance of the MLS model, with a higher $R^{2}$ and lower RMSE values than the MARS approximation. Therefore, the surrogate-based optimization is performed with the MLS model.

\subsection{Single-objective optimization results}

The first strategy applied was a single-objective optimization with the $S E A$ as the objective function. This was performed both unconstrained and constrained with a peak force limit of $250 \mathrm{kN}$, as shown in table 7 . With the design variables given by the algorithm for the optimum designs using the surrogate model, new models were calculated to check for consistency in the results, obtaining errors between the surrogate model and the FEA below 5\%.

When no maximum force limit is specified, the optimum design tends to the highest thicknesses and cell size, with minor changes from the original cell shape. Compared 


\begin{tabular}{lc}
\hline Source & Impact velocity $(\mathrm{m} / \mathrm{s})$ \\
\hline Zou et al. [19] & 6.67 \\
Heimbs et al. [17] & 6.80 \\
Zheng et al. [8] & 7.00 \\
Meng et al. [10] & 7.40 \\
Fasanella et al. [38] & 7.74 \\
Adams and Lankarani [39] & 9.00 \\
Adams et al. [40] & 9.15 \\
Jackson et al. [41] & 9.45 \\
Ren and Xiang [18] & 24.5 \\
\hline Mean & 9.75 \\
\hline
\end{tabular}

Table 5: Experiment and impact velocity correlation for FEM simulations.

\begin{tabular}{llcrl}
\hline Method & Metric & $R^{2}$ & \multicolumn{2}{c}{ RMSE } \\
\hline \multirow{4}{*}{ MLS } & $m$ & 0.9985 & 0.1307 & $\mathrm{~kg}$ \\
& $E_{a}$ & 0.9618 & 19.8514 & $\mathrm{~kJ}$ \\
& $P_{\text {peak }}$ & 0.9903 & 70.4558 & $\mathrm{kN}$ \\
& $S E A$ & 0.8789 & 7.0793 & $\mathrm{~kJ} / \mathrm{kg}$ \\
\hline \multirow{4}{*}{ MARS } & $m$ & 0.9938 & 0.1398 & $\mathrm{~kg}$ \\
& $E_{a}$ & 0.8127 & 21.0837 & $\mathrm{~kJ}$ \\
& $P_{\text {peak }}$ & 0.9367 & 81.2864 & $\mathrm{kN}$ \\
& $S E A$ & 0.2026 & 6.8470 & $\mathrm{~kJ} / \mathrm{kg}$ \\
\hline
\end{tabular}

Table 6: $R^{2}$ and RMSE values for the different metrics of two surrogate models.

with the initial design, the $S E A$ is increased by $136.67 \%$, but also harming the $P_{\text {peak }}$ function by a significant $46.14 \%$. Still, the much smaller effect on the $P_{\text {peak }}$ than on the $S E A$ shows the advantage of this design over the initial specimen.

Given the importance of the $P_{\text {peak }}$ function, a peak force limit value was set to 250 $\mathrm{kN}$. The result is a specimen which betters the initial design's $S E A$ by $65.34 \%$ while maintaining the constraint and reducing $P_{\text {peak }}$ by $18.89 \%$. The resulting cell shape $(\mathrm{S}=0.27)$ differs noticeably from the regular hexagon, as depicted in fig. 9.

In order to identify to which extent was the size optimization beneficial, the optimization was also run fixing the cell with a regular hexagonal shape, thus eliminating the design variable from the optimization. The results show a $S E A$ value $16.30 \%$ higher for the non-regular honeycomb cell shape for an equal peak force constraint.

Furthermore, fig. 10 depicts the objective function value evolution for the constrained optimization as the algorithm performs function evaluations. Starting from a value slightly over $25 \mathrm{~kJ} / \mathrm{kg}$, the $S E A$ increases until the 1000th evaluation, where the objective function value is already near the global optimum. The objective function 


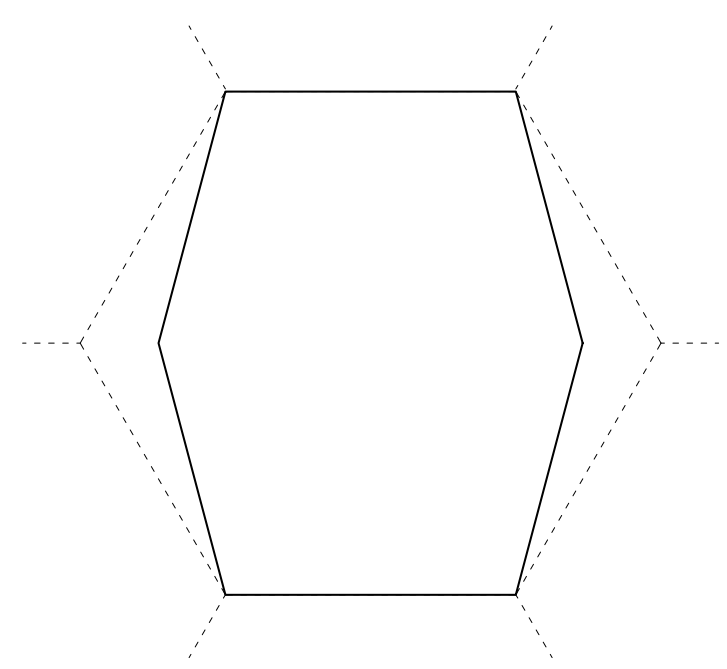

Figure 9: Cell shape of the optimum for the single-objective constrained optimization.

reaches its maximum at approximately the 2500th evaluation, thus justifying the use of surrogate models throughout this investigation.

\begin{tabular}{|c|c|c|c|c|c|c|}
\hline \multirow[t]{2}{*}{ Model } & \multicolumn{4}{|c|}{$\begin{array}{c}\text { Design } \\
\text { variables }(\mathrm{mm})\end{array}$} & \multirow{2}{*}{$\begin{array}{l}\text { Objective } \\
\text { function } \\
S E A(\mathrm{~kJ})\end{array}$} & \multirow{2}{*}{$\begin{array}{l}\text { Constraint } \\
P_{\text {peak }}(\mathrm{kN})\end{array}$} \\
\hline & $\mathrm{L} 1$ & $\mathrm{~T} 1$ & $\mathrm{~T} 2$ & $S$ & & \\
\hline Initial & 6.50 & 1.35 & 1.00 & 0.00 & 15.58 & 307.77 \\
\hline Unconstrained & 14.27 & 2.99 & 2.55 & 0.07 & 36.88 & 449.76 \\
\hline \multirow{2}{*}{ Constrained } & 14.24 & 2.17 & 1.43 & 0.27 & 25.76 & 248.89 \\
\hline & 14.27 & 1.98 & 1.46 & 0.00 & 22.15 & 241.95 \\
\hline
\end{tabular}

Table 7: Single-objective optimization results.

\subsection{Multi-objective optimization results}

The first multi-objective optimization performed to the surrogate model yields a Pareto frontier opposing $S E A$ and $P_{\text {peak }}$, depicted in fig. 13.

The $S E A$ - $P_{\text {peak }}$ frontier has been tabulated with some representative points in table 8. For low $S E A$ and $P_{\text {peak }}$ values, the specimen tends to low thicknesses of both components, with a large cell size and a significant shape variation compared to the regular hexagon. The trend observed as the objective function values increase is that of reducing the cell size, increasing the aluminum thickness and changing the cell towards the regular hexagonal shape. The performance of these specimens is remarkably convenient, since the $S E A$ is significantly improved with little effect on the $P_{\text {peak }}$. However, the opposite effect occurs after the $26 \mathrm{~kJ} / \mathrm{kg}$ threshold, when the honeycomb thickness 


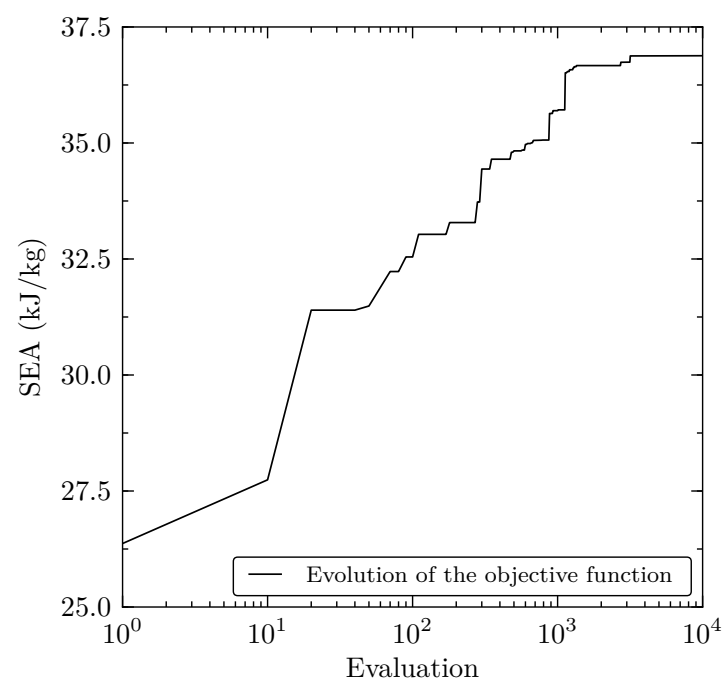

Figure 10: Objective function evolution.

increase noticeably harms the peak force, which had been kept below $250 \mathrm{kN}$. The right end of the Pareto front has again a lower slope, as the cell size and both thicknesses increase, and the cell shape is also slightly affected. The baseline model is strongly dominated by the results on the Pareto front, with designs that reduce its peak force by over $55 \%$ or increase the SEA up to $65 \%$.

\begin{tabular}{rcccccc}
\hline \multicolumn{3}{c}{ Design variables $(\mathrm{mm})$} & & \multicolumn{2}{c}{ Objective functions } \\
\cline { 1 - 2 } \cline { 6 - 7 } $\mathrm{L} 1$ & $\mathrm{~T} 1$ & $\mathrm{~T} 2$ & $\mathrm{~S}$ & & SEA $(\mathrm{kJ} / \mathrm{kg})$ & $P_{\text {peak }}(\mathrm{kN})$ \\
\hline 13.51 & 1.25 & 1.02 & 0.31 & & 14.39 & 135.63 \\
12.51 & 1.40 & 1.02 & 0.17 & & 17.41 & 165.35 \\
11.40 & 1.82 & 1.08 & 0.02 & & 24.44 & 199.22 \\
9.55 & 1.82 & 1.12 & 0.03 & & 25.69 & 240.55 \\
12.66 & 2.17 & 2.00 & 0.04 & & 26.16 & 377.99 \\
14.19 & 2.97 & 1.69 & 0.08 & & 34.12 & 418.32 \\
12.55 & 2.99 & 2.48 & 0.10 & & 35.18 & 508.42 \\
\hline
\end{tabular}

Table 8: Points from $S E A-P_{\text {peak }}$ Pareto front.

The Pareto front from fig. 14 shows the trade-off between mass and energy absorbed, with some of its representative values listed in table 9. The evolution of the variables shows greater mass and energy absorbed as the cell size decreases and the thicknesses increase. The cell shape, always a non-regular hexagon, takes diverse values of $S$, ranging between 0.17 and 0.39 for the greater part of the design space. The section of the frontier for mass values below $5.5 \mathrm{~kg}$ has a pseudo-linear trend, main- 


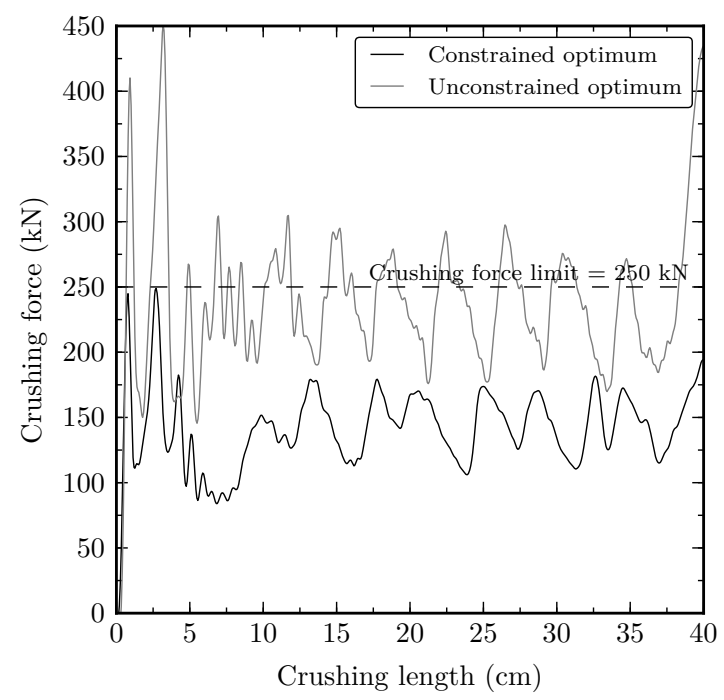

Figure 11: Force - displacement curves of the unconstrained and constrained optima.

taining a proportion between the energy absorption and the mass increase. In the last part of the front the utmost energy absorption is achieved with a hourglass cell shape, but significantly increasing the mass of the specimen. It is also remarkable the progress of the $S E A$, used in this case as an indicator. The highest values, in the vicinity of 31 $\mathrm{kJ} / \mathrm{kg}$, are obtained towards the middle and right end of the Pareto front, always with modified cell shapes, proving that the regular hexagonal honeycomb shape is not the most advantageous for these load cases.

\begin{tabular}{|c|c|c|c|c|c|c|}
\hline \multicolumn{4}{|c|}{ Design variables (mm) } & \multicolumn{2}{|c|}{ Objective functions } & \multirow{2}{*}{$\frac{\text { Indicator }}{S E A(\mathrm{~kJ} / \mathrm{kg})}$} \\
\hline L1 & $\mathrm{T} 1$ & $\mathrm{~T} 2$ & $\mathrm{~S}$ & $\mathrm{~m}(\mathrm{~kg})$ & $E_{a}(\mathrm{~kJ})$ & \\
\hline 12.55 & 1.44 & 1.02 & 0.31 & 1.74 & 34.96 & 20.09 \\
\hline 12.58 & 2.23 & 1.12 & 0.17 & 2.12 & 55.40 & 26.13 \\
\hline 11.40 & 2.99 & 1.02 & 0.30 & 2.58 & 79.63 & 30.86 \\
\hline 6.47 & 2.97 & 1.02 & 0.35 & 3.41 & 106.31 & 31.18 \\
\hline 6.25 & 2.99 & 1.39 & 0.39 & 4.15 & 128.51 & 30.97 \\
\hline 6.44 & 2.97 & 2.82 & 0.18 & 5.64 & 167.80 & 29.75 \\
\hline 6.44 & 2.44 & 2.82 & 0.74 & 8.17 & 187.44 & 22.94 \\
\hline
\end{tabular}

Table 9: Points from mass $-E_{a}$ Pareto front.

\section{Conclusions}

This research focuses on the crashworthiness optimization and improvement of a standard strut located on the lower half of an aircraft's fuselage. The structure has 


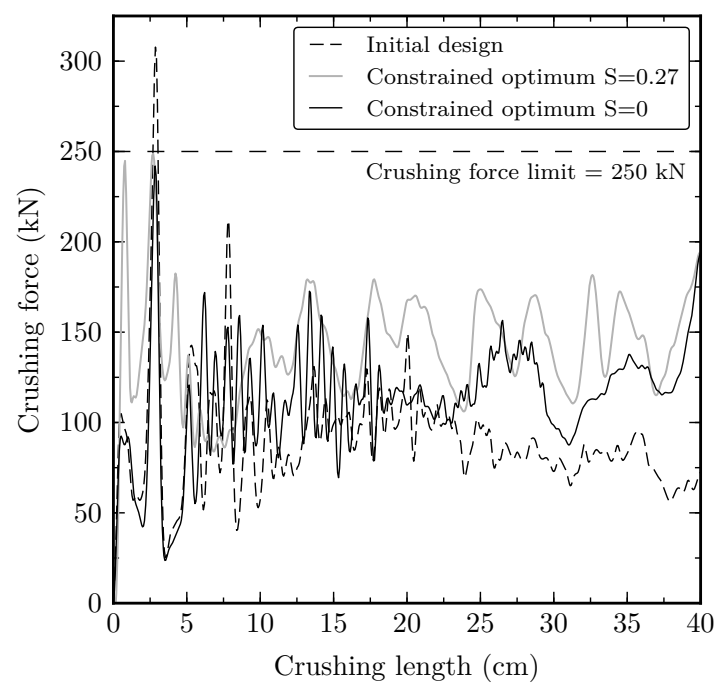

Figure 12: Force - displacement curves of the initial design constrained optima.

been upgraded by adding a GFRP-honeycomb inner reinforcement, with variable thicknesses for the aluminum and the GFRP as well as the shape and size of the cell.

A Moving Least Squares surrogate model is built and subjected to single- and multiobjective optimization with four objective functions: mass, energy absorbed, peak force and the specific energy absorption ratio (SEA). The accuracy of the $R^{2}$ metric is over 0.96 for the first three metrics and 0.87 for the last function.

An unconstrained single-objective optimization of the energy absorbed is first applied, obtaining a value much higher than a baseline design but with an also higher peak force. The constrained optimization with a force limit of $250 \mathrm{kN}$ also improves the initial design, but without surpassing the specified maximum force. This optimized model is characterized by a significantly modified non-regular hexagonal cell shape, which betters a specimen with a regular hexagon and a similar peak force constraint.

Two different multi-objective optimizations have been performed yielding their respective Pareto frontiers. The SEA - peak force front shows that, for low $S E A$ values, the maximum force is kept. However, for higher $S E A$ values the peak force increases boldly, as the GFRP thickness is enlarged to meet with the required energy absorption value. The cell-shape variable takes values proximal to zero, thus being almost a regular hexagon.

The energy absorbed - mass Pareto front behaves pseudo-linearly for designs with a low mass. The cell shape varies significantly, and the $S E A$ values for the points reach their highest values. After that, the frontier's slope - and consequently, the $S E A$ - decreases. At this point, the thicknesses from the GFRP and the aluminum are near their maximum, and the cell, now with an hourglass shape, is close to the lowest specified boundary.

An overall conclusion on the multi-objective optimized results shows a remarkable 


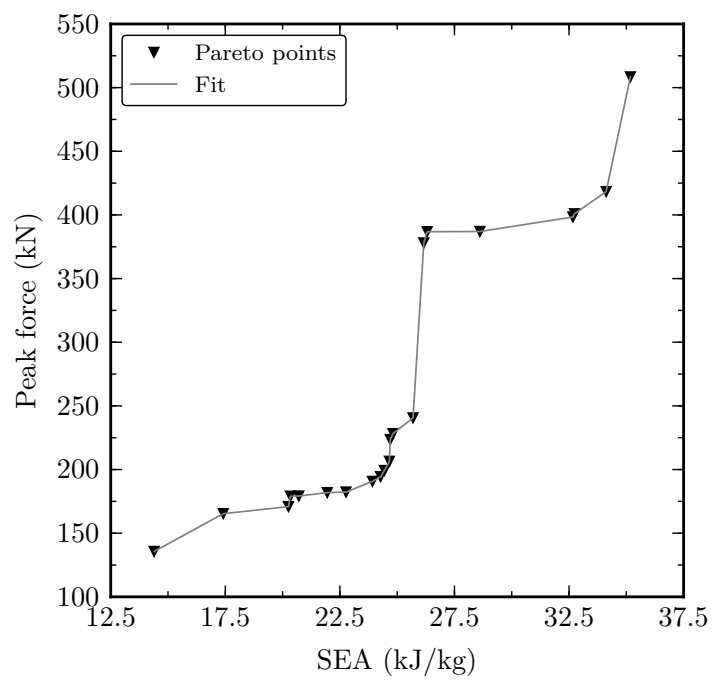

Figure 13: SEA - $P_{\text {peak }}$ Pareto front.

improvement when compared to the initial design. The peak force can be reduced by over 55\% with a similar specific energy absorption, and the specific energy absorption increased by $65 \%$ with no harm on the maximum force.

\section{Acknowledgements}

The research leading to these results has received funding from the Spanish Goverment (Ministerio de Economía y Competitividad) under grant agreement DPI201341893-R. The authors fully acknowledge the support received. 


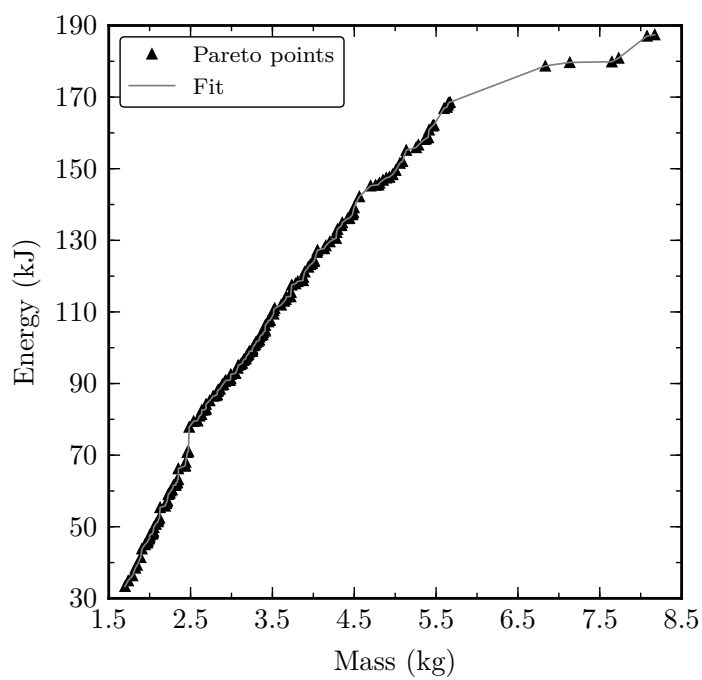

Figure 14: Mass - $E_{a}$ Pareto front. 


\section{References}

[1] F De Florio. Airworthiness: An Introduction to Aircraft Certification. Oxford, 2006.

[2] S. Georgiadis, A.J. Gunnion, R.S. Thomson, and B.K. Cartwright. Bird-strike simulation for certification of the boeing 787 composite moveable trailing edge. Composite Structures, 86(1-3):258-268, 2008.

[3] M. Guida, F. Marulo, M. Meo, A. Grimaldi, and G. Olivares. Sph - lagrangian study of bird impact on leading edge wing. Composite Structures, 93(3):10601071, 2011.

[4] E. L. Fasanella, K. E. Jackson, K. H. Lyle, C. E. Sparks, and A. K. Sareen. Multiterrain impact tests and simulations of an energy absorbing fuselage section. Journal of the American Helicopter Society, 52(2), 2007.

[5] E.G. Candy, N.E. Kirk, and P.J. Murrell. Airframe water impact analysis. International Journal of Crashworthiness, 5(1):51-62, 2000.

[6] Sebastian Heimbs. Energy absorption in aircraft structures. International Workshop on Hydraulic Equipment and Support Systems for Mining, 2012.

[7] C. M. Kindervater and H. Georgi. Composite strength and energy absorption as an aspect of structural crash resistance. Structural Crashworthiness and Failure, 1993.

[8] Jianqiang Zheng, Jinwu Xiang, Zhangping Luo, and Yiru Ren. Crashworthiness design of transport aircraft subfloor using polymer foams. International Journal of Crashworthiness, 16(4):375-383, September 2011.

[9] S Heimbs, T Mehrens, P Middendorf, M Maier, and A Schumacher. Numerical Determination of the Nonlinear Effective Mechanical Properties of Folded Core Structures for Aircraft Sandwich Panels. 6th European LS-DYNA Users' Conference, pages 1-14, February 2011.

[10] F X Meng, Q Zhou, and J L Yang. Improvement of crashworthiness behaviour for simplified structural models of aircraft fuselage. International Journal of Crashworthiness, 14(1):83-97, February 2009.

[11] M. B. Woodson, E. R. Johnson, and R. T. Haftka. Optimal design of composite fuselage frames for crashworthiness. International Journal of Crashworthiness, 1996.

[12] F. Laurin and A.J. Vizzini. Energy absorption of sandwich panels with compositereinforced foam core. Journal of Sandwich Structures and Materials, 7(2):113132, 2005.

[13] K.H. Lyle, A.E. Stockwell, and R.C. Hardy. Application of probability methods to assess airframe crash modeling uncertainty. Journal of Aircraft, 44(5):15681573, 2007. doi: 10.2514/1.27722. 
[14] I. Kumakura, M. Minegishi, and K. Iwasaki. Impact simulation of simplified structural models of aircraft fuselage. SAE Technical Papers, 2000. doi: 10.4271/2000-01-5586.

[15] H. Shoji, M. Minegishi, and T. Aoki. Impact characteristics estimation of channel section short column under axial impact load. Collection of Technical Papers - AIAA/ASME/ASCE/AHS/ASC Structures, Structural Dynamics and Materials Conference, 4:3967-3976, 2007.

[16] Yiru Ren and Jinwu Xiang. A comparative study of the crashworthiness of civil aircraft with different strut configurations. International Journal of Crashworthiness, 15(3):321-330, July 2010.

[17] S. Heimbs, F. Strobl, and P. Middendorf. Integration of a composite crash absorber in aircraft fuselage vertical struts. International Journal of Vehicle Structures and Systems, 2011.

[18] Yiru Ren and Jinwu Xiang. The crashworthiness of civil aircraft using different quadrangular tubes as cabin-floor struts. International Journal of Crashworthiness, 16(3):253-262, June 2011.

[19] Tianchun Zou, Haolei Mou, and Zhenyu Feng. Research on Effects of Oblique Struts on Crashworthiness of Composite Fuselage Sections. Journal of Aircraft, 49(6):2059-2063, November 2012.

[20] H.-W. Song, Z.-M. Wan, Z.-M. Xie, and X.-W. Du. Axial impact behavior and energy absorption efficiency of composite wrapped metal tubes. International Journal of Impact Engineering, 24(4):385-401, 2000.

[21] J Paz, J Díaz, L Romera, and M Costas. Crushing analysis and multi-objective crashworthiness optimization of GFRP honeycomb-filled energy absorption devices. Finite Elements in Analysis and Design, 91:30-39, November 2014.

[22] M. Costas, J. Díaz, L.E. Romera, S. Hernández, and A. Tielas. Static and dynamic axial crushing analysis of car frontal impact hybrid absorbers. International Journal of Impact Engineering, 62:166-181, 2013.

[23] S. Hou, Q. Li, S. Long, X. Yang, and W. Li. Design optimization of regular hexagonal thin-walled columns with crashworthiness criteria. Finite Elements in Analysis and Design, 43(6-7):555-565, 2007.

[24] L. Aktay, A.F. Johnson, and B.-H. Kröplin. Numerical modelling of honeycomb core crush behaviour. Engineering Fracture Mechanics, 75(9):2616-2630, 2008.

[25] H. Yin, G. Wen, and N. Gan. Crashworthiness design for honeycomb structures under axial dynamic loading. International Journal of Computational Methods, 8(4):863-877, 2011.

[26] W Miller, C W Smith, and K E Evans. Honeycomb cores with enhanced buckling strength. Composite Structures, 93(3):1072-1077, 2011. 
[27] Zhenyu Xue and John W Hutchinson. Crush dynamics of square honeycomb sandwich cores. International Journal for Numerical Methods in Engineering, 65(13):2221-2245, 2006.

[28] T. Børvik, O.S. Hopperstad, T.. Berstad, and M. Langseth. A computational model of viscoplasticity and ductile damage for impact and penetration. European Journal of Mechanics, A/Solids, 20(5):685-712, 2001.

[29] T. Børvik, O.S. Hopperstad, and K.O. Pedersen. Quasi-brittle fracture during structural impact of aa7075-t651 aluminium plates. International Journal of Impact Engineering, 37(5):537-551, 2010.

[30] BASF. Ultramid (r) A3WG10 BK00564 Polyamide 66. Product information sheet, July 2012.

[31] SAE Standard J211: Instrumentation for Impact Test. Technical report, Safety Test Instrumentation Stds Comm, 1995.

[32] M. Huang. Vehicle Crash Mechanics. CRC Press, New York, 2002.

[33] K. Yamazaki and J. Han. Maximization of the crushing energy absorption of tubes. Structural Optimization, 16(1):37-46, 1998.

[34] X. Gu, G. Sun, G. Li, L. Mao, and Q. Li. A comparative study on multiobjective reliable and robust optimization for crashworthiness design of vehicle structure. Structural and Multidisciplinary Optimization, pages 1-16, 2013.

[35] M. McKay, R. Beckman, and W Conover. A comparison of three methods for selecting values of input variables in the analysis of output from a computer code. Technometrics, 21(2):239-245, 1979.

[36] P. Lancaster and K. Salkauskas. Surfaces generated by moving least squares methods. Mathematics of Computation, 37(155):141-158, 1981.

[37] J. E. Eddy and K. Lewis. Effective generation of pareto sets using genetic programming. Proceedings of ASME Design Engineering Technical Conference, 2001.

[38] E. L. Fasanella, K. E. Jackson, C. E. Sparks, and A. K. Sareen. Water Impact Test and Simulation of a Composite Energy Absorbing Fuselage Section. American Helicopter Society 59th Annual Forum, pages 1-15, November 2011.

[39] A. Adams and H.M. Lankarani. A modern aerospace modeling approach for evaluation of aircraft fuselage crashworthiness. International Journal of Crashworthiness, 8(4):401-413, 2003.

[40] A Adams, C K Thorbole, and H M Lankarani. Scale modelling of aircraft fuselage: an innovative approach to evaluate and improve crashworthiness. International Journal of Crashworthiness, 15(1):71-82, March 2010. 
[41] Karen E Jackson, Edwin L Fasanella, and Sotiris Kellas. Development of a Scale Model Composite Fuselage Concept for Improved Crashworthiness. Journal of Aircraft, 38(1):95-103, January 2001.

[42] ABAQUS 6.13 Documentation. Dassault Systèmes, 2013.

[43] B. Adams, L. Bauman, W. Bohnhoff, K. Dalbey, M. Ebeida, J. Eddy, M. Eldred, P. Hough, K. Hu, J. Jakeman, L. Swiler, and D. Vigil. DAKOTA, A Multilevel Parallel Object-Oriented Framework for Design Optimization, Parameter Estimation, Uncertainty Quantification, and Sensitivity Analysis: Version 6.1.0 User's Manual. Sandia National Laboratories, 2014. 